\title{
CHARACTERISTICS OF TREE STANDS AND THE TASKS OF SILVICULTURAL INTERVENTIONS IN URBAN FORESTS, AS ILLUSTRATED WITH THE EXAMPLE OF SKAŁKI TWARDOWSKIEGO AND GÓRKA PYCHOWICKA RESERVES
}

\author{
Szymon Worek*, Jakub Kaczmarczyk, Wojciech Majka, \\ Maciej Pach, Jerzy Skrzyszewski
}

Department of Ecology and Silviculture, Faculty of Forestry,

University of Agriculture in Krakow, al. 29 Listopada 46, 31-425 Krakow

\section{ABSTRACT}

The objectives of the study included: (i) assessment of wood resources, advanced regeneration and undergrowth; (ii) inventory of tree microhabitats; and (iii) determining the tasks of cutting treatments in the tree stands in areas of Skałki Twardowskiego and Górka Pychowicka reserves, which are attractive from recreational point of view. The work is a case study, and the tasks of silvicultural interventions in the studied objects were determined based on the preferences of the general public as to the appearance of the forest, resulting from the literature on the subject. The paper presents an analysis of the structure of tending cuts that accomplish the following tasks: removal of trees that pose a threat to safety; removal of trees with extremely shortened crowns or trees that are excessively slender; sanitary cuts consisting in the removal of dying trees or leaving them as microhabitats; removal of invasive species such as ash-leaf maple, American bird cherry; positive selection (in favour of valuable, high-quality, rare species); promotion of single-species groups of trees (removal of another species, group release); removal of one trunk in the trees bifurcated in the butt $(\leq 1.3 \mathrm{~m}$ high); reducing density; supporting

*凶 szymon.worek@onet.pl 
the understory trees and advanced regeneration (shaping a diverse structure); conversion cuts; removal of undergrowth and trees from understory, in order to improve visibility, at a distance of $20 \mathrm{~m}$ from footpaths and clearings. In the tree stands dominated by pine in the Skałki Twardowskiego reserve, conversion cuts were dominant. In tree stands with the desired species composition, mainly the specimens with a higher quality of the trunk were promoted, and cuts were suggested leading to differentiation of the structure. Notably, there is also a large share of cuts aimed at improving safety and sanitation. In the tree stands of Górka Pychowicka reserve, the task of promoting groups, and selection promoting rare species, as well as cuts aimed at differentiating the structure dominated. Also, a relatively large share was the cuts promoting specimens with attractive habit traits, and trees with a higher quality of the trunk. An analysis of the interaction of the effect of planned cuts was performed in the context of providing psychological, sociological, economic and aesthetic benefits to urban communities.

\section{KEYWORDS}

forest management $\bullet$ recreation $\bullet$ silviculture $\bullet$ thinning $\bullet$ urban forestry

\section{Introduction}

The concept and specificity of urban forests are associated with socio-economic changes, the effect of which has been (and continues to be) the massive migration of rural population to the cities (Lawrence 1995). Human need for interaction with the natural environment is natural. In densely populated cities, under conditions of increased stress, the demand for places of rest and recreation is growing rapidly (Kaliszewski 2006). This points to the need to focus forest management on the recreational needs of residents, and not (or to a lesser extent) on wood production (Ważyński 2007). This is expressed in the first definition of urban forestry: "the art, science, and technology of managing trees and forest resources in and around urban ecosystems, in order to provide urban communities with psychological, sociological, economic, and aesthetic benefits" (after: Jaszczak et al. 2017). As reported in their study by Jaszczak et al. (2017), depending on the country, the concept of urban forestry may include not only forests, but also: parks, gardens, cemeteries, wooded areas, tall greenery and even individual trees. In Poland, the management of urban forest stands is related to traditional forestry, but more emphasis is placed on social functions, rather than on production (Łonkiewicz 1997, Ważyński 2007, 2011). In this understanding, the forest should remain a forest ecosystem and cannot be transformed into a forest park (Ważyński 2011, Wajchman 2013). It should be emphasized that management in urban forests, due to their functions and location, is of a different nature than in managed forests (Jaszczak and Wajchman 2015).

Kraków's forest cover is $4.3 \%$, and forests occupy an area of $1,370.80$ ha, which corresponds to $19 \mathrm{~m}^{2}$ per capita (BDL 2020). To compare, we can refer to the data included in the review article by Kaliszewski (2006). The author cites research results, indicating that the average share of green areas in selected European cities is, depending 
on the publication, between $18.5 \%$ and about $30 \%$, and the average size of green areas per capita is $104 \mathrm{~m}^{2}$ (from 6 to 7,000 $\mathrm{m}^{2}$ ). The current forest cover of Kraków is low, and hence the selection of methods enabling these forests to fulfil various functions is important. The management of Kraków's forests is the responsibility of the Zarząd Zieleni Miejskiej (Municipal Greenery Management or ZZM) of the city of Kraków, while the simplified forest management plans are developed by external services (PUL 2018). In order to increase forest cover, the managing authority implemented the "Powiatowy program zwiększania lesistości Miasta Krakowa na lata 2018-2040" [District program for increasing the forest cover of the City of Kraków for 2018-2040] (Nalepa et al. 2018). These forests will undergo maintenance treatments in the near future.

The concept of urban forestry implies the necessity to provide society with benefits from the forest that exceed those offered by commercial forests (Johnston 1996). People resting in the forest prefer: older tree stands with vertical layers and irregularly spaced trees; a larger number of tree species and types of stands with age differences between stands. The differentiation of the structure ensures that the form of the forest remains unchanged over time, which is important, among others, for the aesthetics of the forest (Ważyński 2007). People visiting the forest appreciate the presence of tourist infrastructure (Gundersen and Frivold 2008, Edwards et al. 2012, Giergiczny et al. 2015). Visual issues and landscape management play an important role in recreational forest management (Wajchman 2013). Traces of cuts that had been made or the presence of felling residues (debris) are perceived negatively (Gundersen and Frivold 2008, Giergiczny et al. 2015, Eggers et al. 2018). The respondents describe forests that are loosened, with a wider perspective and longer vistas (but not transformed into parks), and with the share of both deciduous and coniferous trees as more attractive (Zając et al. 2008, Gołos 2018). Single trees should have a long, slender and dense crown (Gerstenberg and Hofmann 2016). Preferences regarding the form of the forest depend, among others, on the socio-demographic factors of the inhabitants, with the respondents being dominated by young, better educated, and wealthier people (Grzelak-Kotulska and Hołowiecka 2013). A significant proportion of people living in large cities derive their knowledge of the forest from observing urban forests (Kaliszewski 2006), hence the educational function of the latter is of great importance.

Recognition of biodiversity, including the presence of tree microhabitats, gives an insight into the nature of the forest in terms of regulatory ecosystem services (Kostecka et al. 2012, Larrieu and Cabanettes 2012). On the other hand, the young generation of the tree stand is indicative of the possibilities for preserving the durability of the forest for recreational purposes (Zygmunt et al. 2014).

The objectives of the study included: (i) assessment of wood resources, advanced regeneration and undergrowth; (ii) inventory of tree microhabitats; and (iii) determining the tasks of cutting treatments in the tree stands in areas of Skałki Twardowskiego and Górka Pychowicka reserves, which are attractive from the recreational point of view. 


\section{Methodology}

\section{Research area}

The area covered by the measurements is located in the stands of Skałki Twardowskiego reserve (subdivisions $41 \mathrm{f}$; o; t w; $\mathrm{x} ; \mathrm{y} ; \mathrm{z} ; \mathrm{ax}$ ) and Górka Pychowicka reserve (division 60 a; b; c; d; h). The selected climatic features of Kraków are: average annual temperature $8.8^{\circ} \mathrm{C}$; average temperature in July $18.9^{\circ} \mathrm{C}$; average annual rainfall $680 \mathrm{~mm}$; average length of the growing season 228 days; average length of the frost-free period 176 days. West and south-west winds prevail (Matuszko and Piotrowicz 2018).

The altitude above sea level of the Skałki Twardowskiego reserve area varies within 205-230 m above sea level (Geoportal 2). Skałki Twardowskiego are a horst, structure, built of Jurassic limestones, and cut with distinct fault lines. In the geological structure of Uroczysko Skałki Twardowskiego there are monocline limestone rocks of the Upper Jurassic series, locally covered with chalk marls in the ceiling. A small fragment is covered with Quaternary sands (Rutkowski 1992, Motyka et al. 2003). The soil cover is varied; the southern part is dominated by acidic brown soils, and the northern part is dominated by postmurshic soil. Forest habitat types include upland forest (division 41 in; $x ; y ; z ; a x)$, fresh forest (division $41 \mathrm{o}$ ) and mixed fresh forest (41 f). The forest stands covered by this study were created as a result of afforestation of wastelands and are characterized by a large share of alien species, including black pine (Pinus nigra Arn.) (Dubiel and Szwagrzyk, eds. 2008).

Altitude differences in the area of Górka Pychowicka reserve are 231-247 m above sea level (Geoportal 2). In the analysed area there are also limestone rocks, mainly Jurassic limestones, locally covered with patches of chalk marl (Rutkowski 1992). There are the following soil types: Rendzic Leptosol; Cambic Rendzic Leptosol; Distric Cambisol. The habitat type of forest was defined as fresh upland forest (PUL 2018).

\section{Field work}

A statistical and mathematical method of inventorying forest resources was used, based on temporal sample plots. Plots with an area of 10 acres were arranged in a grid of squares with the side of $75 \mathrm{~m}$. Measurements were made on 31 plots.

The DBH (diameter at breast height, D) of all trees $(\geq 7 \mathrm{~cm})$ was measured with the accuracy down to $0.1 \mathrm{~cm}$, subdivided into species. The height $(H)$ and crown base height $\left(L_{k}\right)$ were measured on three individual specimens from each type of tree with the accuracy down to $0.1 \mathrm{~m}$ using the Haglöf Vertex IV altimeter. For these trees, the following were also determined:

a) quality (straightness) of the lower ( $\leq 5 \mathrm{~m}$ ) section of the trunk. The amount of curvature was taken into account and the number of adventitious shoots. A 7-point scale was used (MacDonald 2009);

b) tree vitality using the VTA method (Szewczyk 2012). A modified 4-point scale was used (4 - tree fully healthy; 3 - tree with partially dying thinner branches in the api- 
cal parts of the crown and/or the presence of pests, 2 - tree with a minimum loss of $50 \%$ in the assimilation apparatus, and/or the presence of pests; 1 - dying tree, $70 \%$ loss in the assimilation apparatus);

c) occurrence of tree microhabitats divided into 8 types according to the classification by Larrieu et al. (2018). For the lower part of the trunk $(\mathrm{H} \leq 5 \mathrm{~m})$ these were forks, and for the lower and upper part of the trunk $(\mathrm{H}>5)$ : cavities; injuries (damaged cortex and exposed sapwood); burls, growths, "devil's brooms" (excrescences); fungi; epiphyte; exudates; or dead branches.

Inside the sample plots, a concentric circle with a radius equalling half the sample plot radius was set up, in which the number and species of advanced regeneration were recorded. The coverage by undergrowth species expressed as a percentage was estimated (IUL 2012).

Cuttings were designated in all layers of the stand, taking into account silvicultural, visual and other criteria (hiking paths, bicycle routes, forest edge). The following categories of cutting tasks were applied:

- trees posing threat to safety (Szewczyk 2012), inclined, with torn root system, decayed, with dead branches in the crown,

- removal of trees with extremely shortened crowns, extremely slender (H/D),

- sanitary cuttings - removing dying trees or leaving them as microhabitats,

- removal of invasive species, e.g. ash-leaf maple, American bird cherry,

- positive selection in favour of valuable species/specimens,

- positive selection in favour of trees in a shape with high aesthetic value,

- positive selection in favour of rare species,

- promotion of mono-species groups of trees (removal of another species, release of a group of trees),

- removal of one trunk in trees that forked in the butt ( $\leq 1.3 \mathrm{~m}$ high),

- reducing density,

- supporting the trees in understory and advanced regeneration (shaping a diverse structure),

- conversion cuts with the intention of introducing shade-tolerant species - such as fir, beech, hornbeam,

- removal of undergrowth, advanced regeneration and understory in order to improve visibility at a distance of 20 metres from footpaths and clearings.

\section{Analysis}

The location and species composition of the future forest stand were the grouping criteria for subdivision into 3 groups of stands: Skałki Twardowskiego (Skałki A) - preservation of the species composition; Górka Pychowicka - preservation of 
the species composition (Górka); Skałki Twardowskiego - conversion of the species composition (Skałki P). Basic characteristics have been defined and subdivided by species: minimum and maximum $\mathrm{DBH}$ of trees $[\mathrm{cm}]$; maximum height $[\mathrm{m}]$; average $\mathrm{DBH}[\mathrm{cm}]$, tree density $\left[\mathrm{pcs} \cdot \mathrm{ha}^{-1}\right]$; and volume $\left[\mathrm{m}^{3} \cdot \mathrm{ha}^{-1}\right]$. To calculate the volume, height curves were plotted and the tables of the volume of standing trees were used (Czuraj 1998). The following indices were calculated: slenderness ratio expressed as the quotient of the height and $\mathrm{DBH}(\mathrm{H} / \mathrm{D})$; live crown ratio as a ratio of crown length and tree height $\left(L_{k} / H\right)$; average vitality; and average quality. In order to characterize the vertical structure in the scale of 10 acres, the division of the stand into the following layers was used: upper $\left(H>2 / 3 H_{\max }\right)$; middle $\left(2 / 3 H_{\max } \geq H \leq 1 / 3 H_{\max }\right)$; and lower $\left(H<1 / 3 H_{\max }\right)$. The biodiversity indicators were calculated, including: number of species; Margalef index (MRI) (Sienkiewicz 2010); Shannonn index (SDHI); and Simpson index (SIDI) (Nagendra 2002).

Shannon (SHDI) and Simpson (SIDI) indices were calculated according to the formulas:

$$
\begin{aligned}
\mathrm{SHDI} & =1-\sum p_{i} \cdot \ln p_{i} \\
\mathrm{SIDI} & =1-\sum p_{i} \cdot p_{i}
\end{aligned}
$$

where: $p_{i}$ is the share (proportion) of the $i$-th tree species in relation to the sum of shares of all tree species in the group of stands.

The Margalef index was calculated according to the formula:

$$
\operatorname{MRI}=(S-1) / \log N
$$

where:

$S$ - the number of all tree species in the stand group,

$N$ - the number of all specimens.

The average number of the advanced regeneration $\left[\mathrm{pcs} \cdot \mathrm{ha}^{-1}\right]$ and the average coverage by undergrowth species [\%] were determined. The occurrence of tree microhabitats was summarized in groups of stands, subdivided into types. In order to characterize the designated cuts, removed trees according to the number [pcs $\cdot \mathrm{ha}^{-1}$ ] and volume $\left[\mathrm{m}^{3} \cdot \mathrm{ha}^{-1}\right]$ were determined as well as intensity of cuts and structure of tasks. The intensity of cuts in relation to the number of trees was calculated as the ratio of the number of trees removed to their number before the cuts were designated, and in the volume share as the ratio of the volume of trees removed to the volume of trees in the stand before the cuts were introduced (Korpel et al. 1991). 


\section{Results}

Characteristics of groups of tree stands

The number of trees ranged from 582 (Skałka A) to 794 pcs · ha ${ }^{-1}$ (Górka). The growing stock ranged from 244 (Górka) to $385 \mathrm{~m}^{3} \cdot \mathrm{ha}^{-1}$ (Skałki P). The species composition in the Skałki A and Górka groups was dominated by sycamore (Acer pseudoplatanus L.) (30\% and 32\%, respectively); and in Skałki P, by black pine (Pinus nigra J.F. Arnold) (66\%). The remaining species in Skałki A were: English oak (Quercus robur L.) - 20\% and linden (Tilia cordata Mill.) - 14\%, in Górka, the pedunculate oak (Q. robur) - 21\%, red oak (Quercus rubra L .) - 16\% and common maple (Acer platanoides L.) - 13\%. The share of other species in the volume was less than $10 \%$. Average DBH ranged from 20.1 $\mathrm{cm}$ (Górka) to $25.1 \mathrm{~cm}$ (Skałki P). In the Skałki P group, pines (Pinus sp.) (32.3-33.2 $\mathrm{cm}$ ) had relatively large dimensions. The specimen with the greatest thickness was the pedunculate oak (Q. robur) in the Skałki A group with a breast height diameter of 71.3 $\mathrm{cm}$, and the tallest tree was the European larch (Larix decidua Mill.) in Górka, being $29.3 \mathrm{~m}$ high. The slenderness ratio was 85 on average, whereas the highest values (116) were found in European beech (Fagus silvatica L.) in Skałki A. The live crown ratio ranged from 35\% in Górka to 39\% in Skałki A. The shortest crowns were found in pines (24\%) in the group of Skałki P. Tree vitality ranged from 3.4 (Skałki P) to 3.7 (Górka). The least vital were pines (2.3) in the Skałki P group. The quality of trees ranged from 2.9 (Skałki A and Skałki P) to 3.6 (Górka) (Table 1).

\section{Vertical and $\mathrm{DBH}$ structure, and indicators of species diversity}

On the 10 acres scale, the two-layer structure predominated. In all groups of stands, the upper layer was most numerous, with the share ranging from $58 \%$ (Skałki P) to $72 \%$ (Górka). Next was the middle layer, which was found to be the most developed in the Skałki P group (37\%). The number of species ranged from 10 (Górka) to 20 (Skałki P). The Skałki P group had the highest indicators of species diversity (apart from SHDI), while the Górka group had the lowest species diversity. The Shannon index showed the highest value (2.02) for Skałki P (Table 2). 


\begin{tabular}{|c|c|c|c|c|c|c|c|c|c|c|c|c|c|c|c|c|c|c|}
\hline \multicolumn{2}{|c|}{ 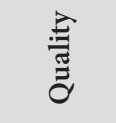 } & \multicolumn{2}{|c|}{$\exists$} & $\stackrel{\vec{i}}{\sim}$ & \multicolumn{2}{|c|}{$\stackrel{m}{i}$} & $\stackrel{\sim}{\sim}$ & $\vec{m}$ & \multicolumn{2}{|c|}{$\stackrel{\circ}{i}$} & $\stackrel{\circ}{\dot{m}}$ & $\stackrel{\circ}{-}$ & $\stackrel{\circ}{i}$ & $\stackrel{\operatorname{Ln}}{i}$ & $\stackrel{\circ}{i}$ & $\stackrel{\circ}{+}$ & $\stackrel{\dot{m}}{\dot{m}}$ & $\hat{i}$ \\
\hline \multicolumn{2}{|c|}{$\stackrel{3}{=}$} & \multicolumn{2}{|c|}{$\grave{m}$} & $\stackrel{\infty}{\infty}$ & \multicolumn{2}{|c|}{$\stackrel{\infty}{\dot{m}}$} & $\stackrel{\circ}{\rightarrow}$ & $\stackrel{\infty}{\oplus}$ & \multicolumn{2}{|c|}{ ì } & $\stackrel{n}{n}$ & $\stackrel{\infty}{\dot{m}}$ & $\stackrel{\circ}{\circ}$ & $\stackrel{\circ}{\rightarrow+}$ & $\stackrel{\circ}{\dot{m}}$ & $\stackrel{m}{m}$ & $\stackrel{\circ}{+}$ & $\stackrel{b}{\dot{m}}$ \\
\hline \multicolumn{2}{|c|}{$\underset{\sim}{\mathbb{N}}$} & \multicolumn{2}{|c|}{ ले } & ले & \multicolumn{2}{|c|}{ m } & r & ๗ँ & \multicolumn{2}{|c|}{ లి } & 우 & $\stackrel{m}{m}$ & in & m & $m$ & $\vec{m}$ & $\stackrel{m}{m}$ & ले \\
\hline \multicolumn{2}{|c|}{ 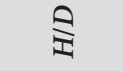 } & \multicolumn{2}{|c|}{$\hat{\wedge}$} & $\stackrel{\circ}{\circ}$ & \multicolumn{2}{|c|}{$\infty$} & $\stackrel{0}{=}$ & in & \multicolumn{2}{|c|}{$N$} & ล̄ & 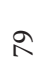 & ถิ & $\stackrel{R}{ }$ & $\stackrel{2}{\wedge}$ & ฉે & $\hat{\sigma}$ & $\stackrel{L}{\infty}$ \\
\hline$\Xi$ & $\Xi$ & \multicolumn{2}{|c|}{$\begin{array}{l}\infty \\
\underset{\sim}{\sim}\end{array}$} & $\hat{\sim}$ & \multicolumn{2}{|c|}{$\stackrel{+}{\stackrel{\sim}{\sim}}$} & $\overrightarrow{ \pm}$ & 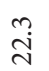 & \multicolumn{2}{|c|}{$\stackrel{\stackrel{H}{N}}{\text { N }}$} & 离 & $\overrightarrow{\dot{d}}$ & $\stackrel{\sim}{\wedge}$ & $\vec{d}$ & $\begin{array}{l}0 \\
\text { ì }\end{array}$ & $\stackrel{\mathscr{n}}{\underset{I}{\nearrow}}$ & $\underset{ \pm}{ت}$ & $\stackrel{+}{\stackrel{\sim}{\sim}}$ \\
\hline$a^{\text {晋 }}$ & ฮี & $\begin{array}{l}1 \\
8\end{array}$ & $\begin{array}{l}0 \\
\text { in }\end{array}$ & $\begin{array}{l}\infty \\
\text { if }\end{array}$ & $\stackrel{m}{i}$ & $\stackrel{\circ}{\ddot{q}}$ & $\stackrel{\vartheta}{\stackrel{\sim}{+}}$ & के & $\begin{array}{l}\infty \\
\dot{f}\end{array}$ & $\underset{\stackrel{+}{v}}{\stackrel{+}{2}}$ & $\stackrel{\circ}{\circ}$ & ळे & $\stackrel{+}{\underset{I}{J}}$ & 官 & $\begin{array}{l}\dot{0} \\
\infty \\
\infty\end{array}$ & $\vec{n}$ & $\stackrel{\circ}{\dot{\sim}}$ & $\stackrel{m}{r}$ \\
\hline$\rho^{\text {䡒 }}$ & ฮี & $\vec{n}$ & $\vec{n}$ & 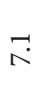 & $\stackrel{\hat{\infty}}{\infty}$ & $\stackrel{\circ}{\mathrm{I}}$ & $\vec{\pi}$ & $\stackrel{\circ}{\infty}$ & $\vec{\Xi}$ & $\stackrel{\circ}{\underset{\sim}{\sim}}$ & $\vec{\Xi}$ & $\begin{array}{l}\infty \\
\infty \\
\infty\end{array}$ & $\ddot{a}$ & $\vec{a}$ & $\stackrel{n}{\stackrel{n}{N}}$ & 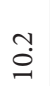 & $\stackrel{\circ}{\stackrel{\sim}{N}}$ & $\vec{r}$ \\
\hline$\theta$ & छี & $\vec{\sim}$ & $\begin{array}{l}\stackrel{+}{\mathrm{J}} \\
\overrightarrow{\mathrm{N}}\end{array}$ & $\stackrel{\infty}{\stackrel{0}{a}}$ & $\stackrel{\text { }}{\stackrel{n}{n}}$ & $\frac{m}{m}$ & $\overrightarrow{\mathrm{I}}$ & $\overrightarrow{\text { จे }}$ & $\stackrel{\circ}{\dot{m}}$ & $\stackrel{\circ}{\stackrel{+}{N}}$ & $\stackrel{n}{\underset{N}{N}}$ & $\hat{\partial}$ & $\hat{o}$ & $\stackrel{\infty}{\stackrel{\infty}{\wedge}}$ & กั & $\stackrel{0}{\ddot{g}}$ & $\stackrel{\sim}{~}$ & $\vec{d}$ \\
\hline & $\alpha^{\circ}$ & Әे & $\stackrel{\sim}{\Upsilon}$ & $\stackrel{\text { mे }}{\vec{v}}$ & & $\underset{\infty}{\infty}$ & $\stackrel{\oplus}{-}$ & $\stackrel{n}{0}$ & $\begin{array}{l}\bullet \\
\dot{b}\end{array}$ & in & $\stackrel{\sim}{\sim}$ & $\stackrel{m}{i}$ & $\overrightarrow{0}$ & $\stackrel{0}{0}$ & $\stackrel{0}{0}$ & $\overrightarrow{0}$ & $\overrightarrow{0}$ & $\stackrel{0}{\dot{8}}$ \\
\hline & $\stackrel{m}{\exists}$ & $\begin{array}{l}\mathbb{b} \\
+ \\
+\end{array}$ & $\begin{array}{l}\hat{b} \\
\stackrel{i}{v}\end{array}$ & $\begin{array}{l}\hat{\infty} \\
\stackrel{\leftrightarrow}{+}\end{array}$ & $\begin{array}{l}\hat{\infty} \\
\text { î }\end{array}$ & $\begin{array}{l}\stackrel{2}{0} \\
\stackrel{\leftrightarrow}{i}\end{array}$ & $\begin{array}{l}\stackrel{m}{+} \\
\stackrel{+}{*}\end{array}$ & $\begin{array}{l}\hat{n} \\
\text { in } \\
\text { no }\end{array}$ & $\begin{array}{l}\vec{\sigma} \\
\vec{i}\end{array}$ & $\stackrel{g}{\rightarrow}$ & $\begin{array}{l}0 \\
0 \\
\emptyset\end{array}$ & $\stackrel{\Re}{\stackrel{7}{ }}$ & $\overrightarrow{\tilde{o}}$ & $\stackrel{\leftrightarrow}{-}$ & $\stackrel{\text { L̊ }}{\text { in }}$ & $\stackrel{7}{0}$ & तิ & $\begin{array}{l}\text { 7 } \\
\stackrel{0}{m}\end{array}$ \\
\hline & $\alpha^{\circ}$ & $\frac{n}{m}$ & $\stackrel{\circ}{\stackrel{0}{0}}$ & $\stackrel{\vec{\nu}}{\vec{\nu}}$ & $\stackrel{\infty}{\infty}$ & $\stackrel{m}{+}$ & $\stackrel{\circ}{\therefore}$ & $\stackrel{n}{+}$ & $\stackrel{m}{f}$ & $\stackrel{\text { ֻै }}{\circ}$ & $\stackrel{n ?}{\longrightarrow}$ & $\stackrel{n}{\rightarrow}$ & $\hat{0}$ & $\hat{0}$ & $\tilde{?}$ & $\stackrel{n ?}{0}$ & ֻั & $\stackrel{\circ}{\dot{8}}$ \\
\hline & $\begin{array}{l}\frac{\pi}{5} \\
\dot{\tilde{S}} \\
\ddot{g}\end{array}$ & $\underset{\sim}{\infty}$ & $\stackrel{\infty}{\infty}$ & ले & กิ & $\stackrel{\mathscr{\sim}}{\sim}$ & F & $\stackrel{\bullet}{\sim}$ & $\stackrel{\Perp}{\sim}$ & $\neg$ & $a$ & $a$ & + & + & $m$ & $m$ & - & $\underset{\infty}{\infty}$ \\
\hline & & 官 & 安 & نُ & $\stackrel{\dot{0}}{\dot{0}}$ & $\stackrel{\ddot{\sigma}}{\dot{d}}$ & $\begin{array}{l}\dot{\vec{D}} \\
\overrightarrow{\tilde{D}}\end{array}$ & & $\begin{array}{l}\dot{\omega} \\
\sim^{\prime}\end{array}$ & $\ddot{\vec{n}}$ & نَّ & 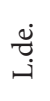 & 㝕 & $\frac{9}{5}$ & 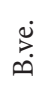 & $\begin{array}{l}\stackrel{\circlearrowright}{ப} \\
\dot{\infty}\end{array}$ & 离 & స్ㅠㅇ \\
\hline & & & & & & & & & & 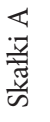 & & & & & & & & \\
\hline
\end{tabular}




\begin{tabular}{|c|c|c|c|c|c|c|c|c|c|c|c|c|c|c|c|c|c|c|}
\hline \multicolumn{2}{|c|}{$\stackrel{m}{+}$} & \multicolumn{2}{|c|}{$\underset{\text { i }}{\text { in }}$} & $\stackrel{\infty}{m}$ & $\widehat{\vec{i}}$ & $\stackrel{n}{\rightarrow}$ & in & $\stackrel{\operatorname{Ln}}{i}$ & $\stackrel{\circ}{\dot{m}}$ & $\begin{array}{l}\dot{r} \\
\dot{m}\end{array}$ & \multicolumn{2}{|c|}{$\stackrel{\infty}{-}$} & \multicolumn{3}{|c|}{$\cong$} & \multicolumn{2}{|c|}{$\stackrel{\circ}{i}$} & \multirow{2}{*}{$\begin{array}{l}\dot{\rho} \\
\dot{m} \\
\dot{m}\end{array}$} \\
\hline & $\dot{0}$ & $i$ & : & $\ddot{r}$ & $\ddot{m}$ & $\stackrel{\infty}{\oplus}$ & $\underset{r}{\ddot{r}}$ & $\stackrel{\circ}{+}$ & $\stackrel{\circ}{+}$ & $\stackrel{m}{m}$ & s & & & $\stackrel{\infty}{\oplus}$ & & 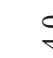 & & \\
\hline & & c & ? & $\stackrel{\operatorname{Ln}}{m}$ & mे & $\stackrel{m}{n}$ & $\stackrel{m}{n}$ & in & $\ddot{m}$ & in & 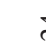 & & & ले & & st & & 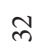 \\
\hline \multicolumn{2}{|c|}{$\triangleright$} & \multicolumn{2}{|c|}{$\alpha$} & $\stackrel{\infty}{\wedge}$ & $\varpi_{\infty}$ & హ్ & $\infty$ & $\stackrel{\circ}{ }$ & $\mathbb{N}$ & $\mathscr{L}$ & 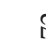 & & \multicolumn{3}{|c|}{$\kappa$} & \multicolumn{2}{|c|}{ ๙ั } & $\curvearrowright$ \\
\hline \multicolumn{2}{|c|}{$\stackrel{\vec{\nu}}{\hat{\nu}}$} & \multicolumn{2}{|c|}{$\begin{array}{l}\text { ம. } \\
\dot{\nu}\end{array}$} & $\begin{array}{l}0 \\
\stackrel{i}{~}\end{array}$ & $\begin{array}{l}\ddot{H} \\
\text { 㟧 }\end{array}$ & $\stackrel{\text { ஸे }}{\text { aे }}$ & ڤે & $\stackrel{+}{\vec{\lambda}}$ & $\stackrel{\sim}{\infty}$ & $\stackrel{m}{\grave{d}}$ & \multicolumn{2}{|c|}{$\vec{d}$} & \multicolumn{3}{|c|}{$\stackrel{\circ}{\stackrel{i}{N}}$} & \multicolumn{2}{|c|}{$\begin{array}{l}\text { : } \\
\stackrel{i}{0}\end{array}$} & $\stackrel{n}{\sim}$ \\
\hline$\vec{n}$ & $\dot{m}$ & $\vec{\infty}$ & $\stackrel{n}{\curvearrowright}$ & $\vec{m}$ & กั. & $\ddot{~}$ & $\begin{array}{l}\text { n̊. } \\
\text { }\end{array}$ & $\stackrel{m}{m}$ & $\stackrel{\sim}{n}$ & ָ̊ & gे & $\vec{f}$ & $\begin{array}{l}0 \\
\infty \\
\infty\end{array}$ & $\begin{array}{l}n \\
b \\
\mathscr{f}\end{array}$ & $\begin{array}{l}\stackrel{0}{\sim} \\
\ddot{\sim}\end{array}$ & $\stackrel{\ddot{m}}{\dot{m}}$ & 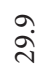 & $\stackrel{\infty}{\ddot{f}}$ \\
\hline$\vec{\Gamma}$ & $\vec{r}$ & $\vec{\pi}$ & $\stackrel{t}{r}$ & $\stackrel{\infty}{\wedge}$ & $\ddot{a}$ & aे & $\stackrel{\sim}{=}$ & $\stackrel{\sim}{2}$ & 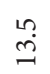 & $\vec{n}$ & $\stackrel{\circ}{\exists}$ & $\overrightarrow{\mathrm{I}}$ & 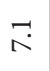 & $\vec{n}$ & $\stackrel{n}{0}$ & $\vec{\therefore}$ & 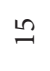 & $\tilde{a}$ \\
\hline $\begin{array}{l}0 \\
\infty \\
-1\end{array}$ & 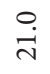 & $\begin{array}{l}n \\
\infty \\
-1\end{array}$ & $\stackrel{9}{\underline{1}}$ & $\stackrel{\not}{\not}$ & $\stackrel{+}{\stackrel{\sim}{N}}$ & $\begin{array}{l}0 \\
\stackrel{+}{+}\end{array}$ & $\begin{array}{l}0 \\
\stackrel{+}{+}\end{array}$ & $\hat{\imath}$ & 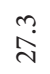 & $\overrightarrow{\text { d }}$ & $\stackrel{m}{\pi}$ & $\stackrel{\sim}{m}$ & $\stackrel{\circ}{0}$ & $\stackrel{m}{\sim}$ & $\stackrel{\circ}{\circ}$ & $\stackrel{m}{=}$ & $\vec{\sim}$ & $\begin{array}{l}\text { nִ } \\
\text { ֶे }\end{array}$ \\
\hline$\stackrel{n}{m}$ & $\begin{array}{l}\infty \\
\mathfrak{I}\end{array}$ & $\stackrel{+H}{\vec{i}}$ & $\begin{array}{l}\infty \\
\stackrel{\varphi}{~}\end{array}$ & $\stackrel{\sharp}{\sim}$ & $\hat{n}$ & $\underset{i}{\stackrel{r}{i}}$ & $\grave{o}$ & $\stackrel{\sim}{m}$ & $\stackrel{1}{0}$ & $\begin{array}{l}\text { ¿ } \\
\dot{8}\end{array}$ & $\begin{array}{l}n \\
\tilde{n}\end{array}$ & $\underset{\infty}{+}$ & $\ddot{a}$ & $\hat{m}$ & $\stackrel{6}{-}$ & $\stackrel{9}{-}$ & $\overrightarrow{0}$ & $\stackrel{b}{\dot{r}}$ \\
\hline$\stackrel{\overbrace{}}{\pi}$ & बे & $\begin{array}{l}\infty \\
\infty \\
\infty \\
i\end{array}$ & $\underset{\infty}{\stackrel{9}{0}}$ & $\begin{array}{l}0 \\
\infty \\
\varrho \\
0\end{array}$ & $\begin{array}{l}n \\
\hat{n} \\
\infty \\
+\rightarrow\end{array}$ & $\begin{array}{l}\infty \\
\infty \\
\text { in }\end{array}$ & 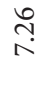 & $\stackrel{\hat{o}}{i}$ & $\underset{\sim}{\stackrel{H}{\sim}}$ & $\begin{array}{l}\text { in } \\
\text { In } \\
\text { J }\end{array}$ & $\begin{array}{l}\text { ָे } \\
\text { ָై }\end{array}$ & $\begin{array}{l}\text { 命 } \\
\text { in }\end{array}$ & $\begin{array}{l}\hat{\imath} \\
\stackrel{b}{0} \\
\text { no }\end{array}$ & $\begin{array}{l}\underset{J}{ \pm} \\
\underset{J}{J}\end{array}$ & $\stackrel{\overbrace{}}{0}$ & $\stackrel{\overbrace{}}{\curvearrowright}$ & గొ & $\begin{array}{l}\stackrel{n}{O} \\
\underset{\sim}{+}\end{array}$ \\
\hline$\stackrel{\stackrel{p}{q}}{\circ}$ & $\stackrel{\sim}{\stackrel{n}{\sim}}$ & $\stackrel{\infty}{a}$ & $\hat{a}$ & $\stackrel{\text { Nִ }}{0}$ & $\tilde{u}$ & $\stackrel{m}{i}$ & $\stackrel{\sharp}{0}$ & $\stackrel{9}{-}$ & $\stackrel{m}{0}$ & $\stackrel{0}{\dot{8}}$ & $\hat{o}$ & 아 & $\begin{array}{l}n \\
\stackrel{i}{0}\end{array}$ & ì & $\stackrel{\circ}{i}$ & $\stackrel{\circ}{m}$ & $\dddot{m}$ & $\stackrel{\circ}{\dot{m}}$ \\
\hline 今े & $\stackrel{\infty}{=}$ & $\vec{\beth}$ & กี & $\infty$ & $\stackrel{\infty}{\wedge}$ & $\curvearrowright$ & $\stackrel{n}{n}$ & $m$ & $N$ & ন্থ & 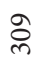 & $\stackrel{\infty}{m}$ & $\stackrel{n}{\Omega}$ & $\stackrel{\sim}{=}$ & $\stackrel{2}{\sim}$ & $\ddot{\sim}$ & N & $\ddot{\sim}$ \\
\hline$\stackrel{\dot{p}}{\dot{4}}$ & نे & $\stackrel{\leftrightarrow}{\circ}$ & $\stackrel{\vec{\Xi}}{\dot{0}}$ & $\stackrel{\dot{0}}{\leftrightarrow}$ & Ф் & ن. & 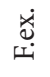 & $\underset{ت}{\stackrel{\vec{\varpi}}{~}}$ & $\frac{\pi}{5}$ & تే & ن. & نُ & $\stackrel{\leftrightarrow}{\stackrel{4}{4}}$ & 安 & نே் & $\frac{\pi}{5}$ & 苂 & 官 \\
\hline & & & & & & & & & $\frac{a}{\frac{\vec{V}}{\vec{*}}}$ & & & & & & & & & \\
\hline
\end{tabular}




\begin{tabular}{|c|c|c|c|c|c|c|c|c|c|c|c|c|c|c|c|}
\hline \multicolumn{2}{|c|}{ 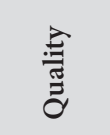 } & $\stackrel{\not H}{*}$ & \multicolumn{2}{|c|}{$\stackrel{n}{m}$} & \multicolumn{3}{|c|}{$\stackrel{b}{+}$} & $\stackrel{H}{+}$ & $\stackrel{\circ}{-}$ & $\stackrel{\sim}{\underset{H}{*}}$ & $\stackrel{\sim}{+}$ & $\stackrel{\circ}{-}$ & $\dot{m}$ & 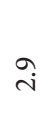 & 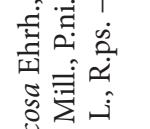 \\
\hline \multicolumn{2}{|c|}{ 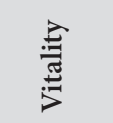 } & $\stackrel{\infty}{\oplus}$ & \multicolumn{2}{|c|}{$\stackrel{\infty}{\oplus}$} & \multicolumn{3}{|c|}{$\stackrel{\infty}{\oplus}$} & $\stackrel{\circ}{\rightarrow}$ & $\stackrel{\bullet}{m}$ & $\stackrel{\circ}{\rightarrow}$ & $\stackrel{\circ}{\leftrightarrow}$ & $\stackrel{\dot{m}}{.}$ & $\stackrel{\circ}{\leftrightarrow}$ & $\stackrel{\ddot{m}}{m}$ & 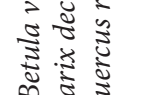 \\
\hline \multicolumn{2}{|c|}{$\underset{v}{\Xi}$} & $\ddot{m}$ & \multicolumn{2}{|c|}{ के } & \multicolumn{3}{|c|}{$\stackrel{\infty}{n}$} & m & $\stackrel{F}{F}$ & 와 & 우 & $\stackrel{\mathscr{n}}{\sim}$ & $m$ & $\stackrel{m}{m}$ & 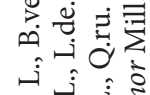 \\
\hline \multicolumn{2}{|c|}{$\stackrel{ి}{\mathrm{I}}$} & $\stackrel{2 n}{\wedge}$ & \multicolumn{2}{|c|}{ 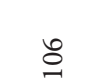 } & \multicolumn{3}{|c|}{$\infty$} & $\infty$ & $\approx$ & $\curvearrowright$ & ठే & in & $\hat{\sigma}$ & $\infty$ & 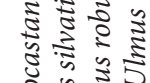 \\
\hline$\Xi^{.}$ & $\Xi$ & $\stackrel{\vartheta}{0}$ & \multicolumn{2}{|c|}{$\stackrel{m}{i}$} & \multicolumn{3}{|c|}{$\stackrel{\infty}{\sim}$} & $\stackrel{+}{\stackrel{+}{i}}$ & $\stackrel{\curvearrowright}{\wedge}$ & $\stackrel{m}{n}$ & $\stackrel{m}{i}$ & $\stackrel{m}{i}$ & $\stackrel{0}{\check{1}}$ & $\vec{d}$ & $\begin{array}{llll}1 & 1 \\
3 & 0 & 0 \\
3 & 0\end{array}$ \\
\hline$a^{\stackrel{.}{g}}$ & छี & $\stackrel{n}{i}$ & $\stackrel{0}{\dot{m}}$ & $\stackrel{\text { ̀े }}{ }$ & $\stackrel{n}{\stackrel{n}{f}}$ & $\stackrel{a}{\sim}$ & $\stackrel{\circ}{\dot{\nu}}$ & $\stackrel{\uparrow}{\infty}$ & $\stackrel{\infty}{\stackrel{\infty}{f}}$ & $\stackrel{m}{n}$ & ì & 官 & $\stackrel{0}{\infty}$ & ڤें & 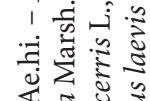 \\
\hline$a^{\frac{5}{a}}$ & छี & $\vec{n}$ & $\stackrel{\sharp !}{N}$ & $\stackrel{\circ}{i}$ & $\stackrel{+}{\leftrightarrows}$ & $\stackrel{0}{\circ}$ & 욤 & $\stackrel{\sim}{\sim}$ & $\stackrel{\sim}{\infty}$ & $\stackrel{a}{\jmath}$ & $\begin{array}{l}\dot{0} \\
\dot{n}\end{array}$ & 官 & $\stackrel{0}{\infty}$ & $\stackrel{\circ}{\wedge}$ & 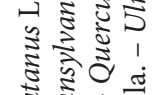 \\
\hline$\theta$ & ฮี & $\stackrel{g}{ \pm}$ & $\stackrel{-1}{\infty}$ & $\grave{\mathfrak{I}}$ & $\underset{+}{+}$ & $\stackrel{\sim}{2}$ & $\stackrel{\circ}{\dot{\nu}}$ & $\stackrel{n}{i}$ & in & $\stackrel{m}{\sharp}$ & $\stackrel{\sim}{\sim}$ & $\stackrel{p}{m}$ & $\stackrel{\infty}{-}$ & $\vec{n}$ & 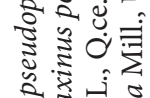 \\
\hline & $d^{0}$ & $\stackrel{n}{0}$ & $\stackrel{0}{0}$ & $\ddot{0}$ & $\grave{o}$ & $\tilde{o}$ & $\ddot{0}$ & $\stackrel{m}{-}$ & $\stackrel{+}{-}$ & ֻุ & $\stackrel{n}{0}$ & ஸุ & $\ddot{0}$ & $\stackrel{\circ}{\dot{8}}$ & 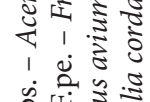 \\
\hline & 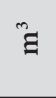 & $\stackrel{\infty}{\stackrel{-}{-}}$ & $\stackrel{m}{i}$ & $\stackrel{n}{\tilde{o}}$ & $\stackrel{m}{m}$ & $\begin{array}{l}\stackrel{0}{0} \\
\vdots\end{array}$ & $\stackrel{\circ}{\circ}$ & $\underset{⿱ ㇒}{ \pm}$ & $\begin{array}{c}m \\
\text { in }\end{array}$ & $\stackrel{8}{\circ}$ & ڤ̊ & 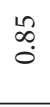 & $\stackrel{\text { ণ̊ }}{0}$ & $\begin{array}{l}\tilde{n} \\
i \\
\infty \\
\infty\end{array}$ & 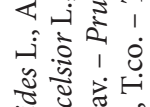 \\
\hline 7 & $d^{0}$ & $\stackrel{\circ}{r}$ & $\stackrel{?}{\longrightarrow}$ & $\stackrel{l}{0}$ & 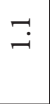 & $\hat{0}$ & $\overrightarrow{0}$ & $\cong$ & $\tilde{0}$ & $\ddot{0}$ & $\dddot{m}$ & $\overrightarrow{0}$ & $\overrightarrow{0}$ & $\stackrel{0}{\dot{8}}$ & 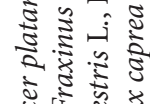 \\
\hline & 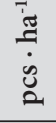 & $\ddot{\sim}$ & $=$ & 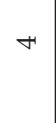 & $\infty$ & in & $\neg$ & $\stackrel{m}{-}$ & + & $m$ & $N$ & - & $\neg$ & lo & 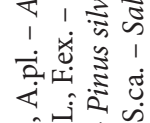 \\
\hline & & 悹 & نجّ & نَ & $\dot{0}$ & $\ddot{\dot{d}}$ & $\stackrel{\overrightarrow{0}}{\ddot{\theta}}$ & 寄 & i் & $\ddot{\tilde{S}}$ & $\stackrel{\dot{8}}{\dot{\leftrightarrow}}$ & 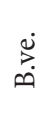 & 苞 & సٓㅠㅇ & 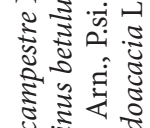 \\
\hline & & & & & & & & $\begin{array}{l}n \\
\frac{\pi}{\pi} \\
\frac{\pi}{n}\end{array}$ & & & & & & & 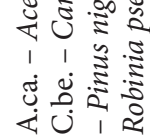 \\
\hline
\end{tabular}


Table 2. Vertical structure and indices of species diversity: Margalef (MRI), Shannon (SHDI) and Simpson (SIDI) determined for tree species

\begin{tabular}{|c|c|c|c|c|}
\hline \multicolumn{2}{|c|}{ Area } & Skałki A & Górka & Skałki P \\
\hline \multirow{4}{*}{ Layer } & Upper [\%] & 69 & 72 & 58 \\
\cline { 2 - 5 } & Middle [\%] & 24 & 27 & 37 \\
\cline { 2 - 5 } & Lower [\%] & 7 & 1 & 5 \\
\hline \multirow{7}{*}{ Index } & Number of species & 16 & 10 & 20 \\
\cline { 2 - 5 } & MRI & 2.36 & 1.35 & 2.41 \\
\cline { 2 - 5 } & SHDI & 2.02 & 1.71 & 1.87 \\
\cline { 2 - 5 } & SIDI & 5.48 & 4.03 & 7.04 \\
\hline
\end{tabular}

Distributions of DBH for the groups Skałki A and Górka took the form of unimodal right-skewed distribution, whereas the distribution for Skałki P was bimodal. In all groups of stands, the lowest thickness class was the most numerous (from 7 to $14.9 \mathrm{~cm}$ ). The share of trees in particular thickness classes decreased with an increase in DBH class in the Skałki A and Górka groups. In the Skałki P group, the second largest class (in terms of share) represented the range from 23 to $30.9 \mathrm{~cm}$ (Fig. 1).

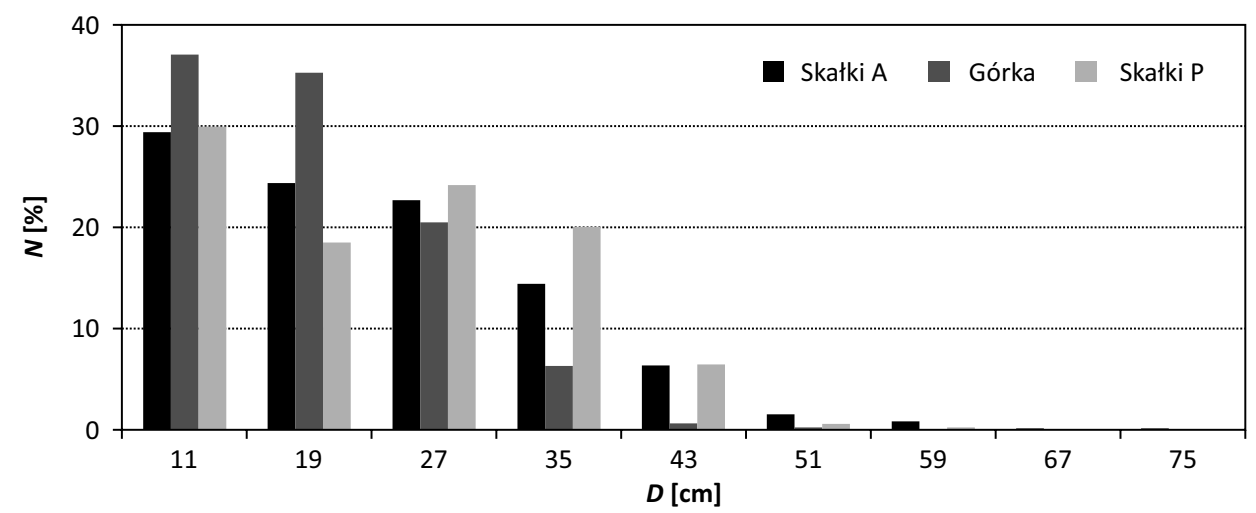

Fig. 1. DBH distribution $(N)$ in groups of tree stands, 8-centimeter classes of diameters $(D)$

Advanced regeneration (saplings)

In the studied groups of stands, the number of saplings ranged from 2,764 to 2,948 pcs. $\cdot \mathrm{ha}^{-1}$. The smallest number of saplings was found in the group in which the conversion cuts were designed. The species composition was dominated by species of maple (Acer spp. L.). In Skałki A, sycamore (A. pseudoplatanus) had a share of 49.7\%, and in Skałki P - 55.7\%. Common maple (A. platanoides) dominated in Górka (86.4\%). 


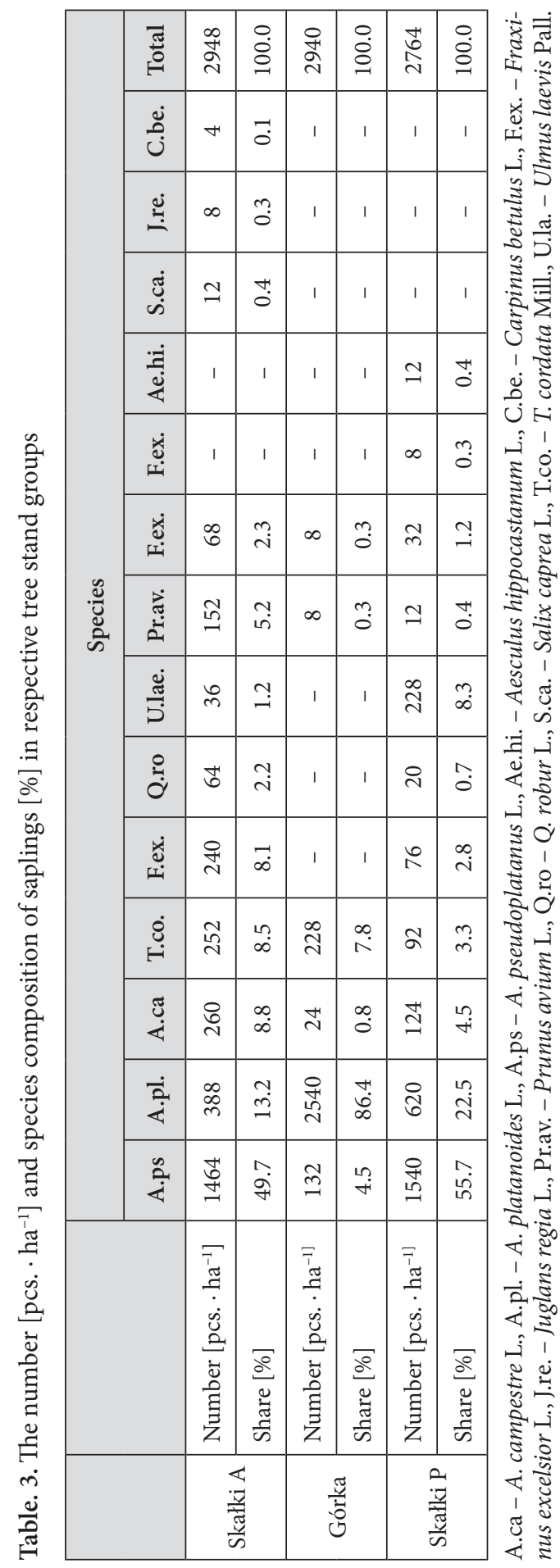


The share of the remaining species was lower. In the groups Skałki A and Skałki P the occurrence of alien species was found. In Skałki A, walnut (Juglans regia L.) was found; in Skałki P, Pennsylvanian ash (Fraxinus pennsylvanica Marsh.) and horse chestnut (Aesculus hippocastanum L.); and in the stands of both groups, the Burgundy oak (Quercus cerris L.) (Table 3).

\section{Undergrowth}

Undergrowth species covered $35-40 \%$ of the area. The greatest coverage was found in Górka (43\%), and the lowest in Skałki P (35\%). The most frequently registered species in both groups of Skałki was elderberry (Sambucus nigra L.), and in Górka, it was dogwood (Cornus sanguinea L.). In addition, the American bird cherry (Prunus serotina Ehrh.) occurred in both Skałki groups (Table 4).

Table 4. Undergrowth cover

\begin{tabular}{|c|l|c|}
\hline Group & \multicolumn{1}{|c|}{ Species } & Cover [\%] \\
\hline Skałki A & $\begin{array}{l}\text { Elder (Sambucus nigra L.), dogwood (Cornus sanguinea L.), hawthorn } \\
\text { (Crataegus monogyna Jacq.), bird cherry (Prunus serotina Ehrh), } \\
\text { buckthorn (Rhamnus cathartica L.), hazel (Corylus avellana L.), privet } \\
\text { (Ligustrum vulgare L.) }\end{array}$ & 40 \\
\hline Górka & $\begin{array}{l}\text { Elder (S. nigra L.), dogwood (C. sanguinea L), buckthorn (R. cathartica L.), } \\
\text { hawthorn (C. monogyna Jacq.), blackthorn (Prunus spinosa L.) }\end{array}$ & 43 \\
\hline Skałki P & $\begin{array}{l}\text { Elder (S. nigra L.), dogwood (C. sanguinea L.), hawthorn (C. monogyna } \\
\text { Jacq.), bird chery (P. serotina Ehrh), guelder-rose (Viburnum opulus L.) } \\
\text { buckthorn (R. cathartica L.), }\end{array}$ & 35 \\
\hline
\end{tabular}

\section{Tree microhabitats}

The number of registered tree microhabitats ranged from 74 pcs. $\cdot$ ha $^{-1}$ (in Skałki A) to 116 pcs. $\cdot$ ha $^{-1}$ (in Skałki P). The most frequently recorded microhabitats in the Skałki A group of stands were forks (19 pcs. $\left.\cdot \mathrm{ha}^{-1}\right)$; in group Skałki A, epiphytes in the lower part of the trunk ( 21 pcs. $\cdot$ ha $\left.^{-1}\right)$; and in the group of Skałki P stands, dead branches in the crown $\left(26\right.$ pcs. $\cdot$ ha $\left.^{-1}\right)$. The last group showed a large number of forks $\left(25\right.$ pcs. $\cdot$ ha $\left.^{-1}\right)$ (Fig. 2).

\section{Cuts}

In the established groups of stands, between 100 trees (in Skałki A) and 152 trees (in Skałki P) per 1 ha were designated for removal. The volume of tree removed ranged from 25.8 (Górka) to 96.7 (Skałki P) $\mathrm{m}^{3} \cdot \mathrm{ha}^{-1}$. The intensity of the cuts, when measured by the volume of the removed trees, ranged from $11.3 \%$ (Górka) to $25.2 \%$ (Skałki P), and when 


\begin{tabular}{|c|c|c|c|c|c|c|c|c|c|c|c|c|c|c|c|c|}
\hline \multirow{6}{*}{$\begin{array}{l}0 \\
\frac{7}{3} \\
\frac{\pi}{4}\end{array}$} & \multirow{2}{*}{ 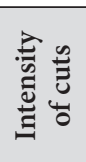 } & $\vec{\lambda}^{2}$ & $\partial^{\circ}$ & $\stackrel{\circ}{\circ}$ & $\stackrel{0}{\circ}$ & $\grave{o}$ & $\stackrel{\infty}{-}$ & $\stackrel{\circ}{-}$ & $\hat{o}$ & $\stackrel{\circ}{\circ}$ & $\overrightarrow{0}$ & $\stackrel{n}{0}$ & $\stackrel{\circ}{\circ}$ & $\begin{array}{l}0 \\
\stackrel{1}{\Delta}\end{array}$ & 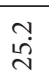 & 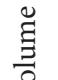 \\
\hline & & $\begin{array}{l}z \\
z\end{array}$ & $\partial^{\circ}$ & $\stackrel{\sim}{-}$ & $\stackrel{10}{0}$ & $\stackrel{\circ}{-}$ & $\stackrel{\infty}{i}$ & $\stackrel{\circ}{-}$ & $\exists$ & $\overrightarrow{0}$ & $\stackrel{1}{0}$ & $\stackrel{+}{\circ}$ & $\stackrel{1}{0}$ & $\exists$ & กิ & $\begin{array}{l}7 \\
1 \\
3\end{array}$ \\
\hline & \multirow{4}{*}{ 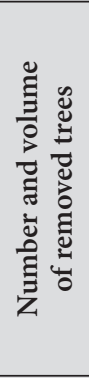 } & \multirow[b]{2}{*}{$\lambda^{=}$} & $\partial^{\circ}$ & ชี & $\stackrel{H}{i}$ & $\hat{m}$ & $\stackrel{N}{N}$ & ભે & $\stackrel{\leftrightarrow}{\mathrm{i}}$ & $\because$ & กั & $\stackrel{\circ}{-}$ & $\stackrel{\sim}{\sim}$ & ดे & 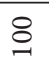 & 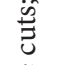 \\
\hline & & & 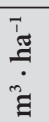 & $\stackrel{\overrightarrow{0}}{0}$ & $\tilde{\overbrace{}}$ & $\vec{n}$ & $\stackrel{n}{6}$ & $\stackrel{\hat{N}}{\hat{n}}$ & $\begin{array}{l}+ \\
\stackrel{+}{+} \\
\stackrel{i}{*}\end{array}$ & $\stackrel{n}{\circ}$ & $\stackrel{\infty}{\stackrel{0}{0}}$ & $\hat{a}$ & 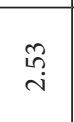 & $\begin{array}{l}\overrightarrow{6} \\
\hat{\sigma}\end{array}$ & $\begin{array}{l}\stackrel{+}{\hat{~}} \\
\text { }\end{array}$ & $\begin{array}{l}\bar{Z} \\
\stackrel{0}{0} \\
\stackrel{0}{0}\end{array}$ \\
\hline & & \multirow[b]{2}{*}{$z^{*}$} & $\partial^{\circ}$ & $\stackrel{0}{0}$ & $\stackrel{+}{i}$ & $\stackrel{\infty}{+}$ & $\stackrel{m}{+}$ & $\stackrel{\infty}{+}$ & $\ddot{H}$ & $\stackrel{0}{0}$ & $\stackrel{+}{i}$ & $\stackrel{\infty}{-}$ & $\stackrel{+}{i}$ & $\overrightarrow{i n}$ & 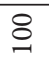 & $\bar{\Xi}$ \\
\hline & & & 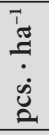 & $a$ & 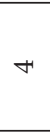 & $\wedge$ & $\underset{\sim}{\sim}$ & $\wedge$ & $\infty$ & - & 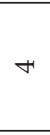 & $m$ & 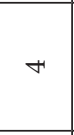 & $\varpi_{\infty}$ & กี & 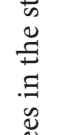 \\
\hline \multirow{6}{*}{ 䙳 } & \multirow{2}{*}{ 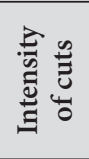 } & 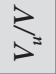 & $\partial^{\circ}$ & $\overrightarrow{0}$ & ก̋ & $\ddot{0}$ & $\stackrel{\circ}{-}$ & $\hat{o}$ & $\stackrel{\circ}{i}$ & $\stackrel{b}{\dot{r}}$ & $\stackrel{H}{0}$ & $\stackrel{?}{\circ}$ & $\begin{array}{l}b \\
i \\
\stackrel{0}{0}\end{array}$ & ' & $\stackrel{n}{=}$ & \\
\hline & & $\begin{array}{l}z \\
z\end{array}$ & $\alpha^{\circ}$ & $\overrightarrow{0}$ & $\stackrel{1}{0}$ & $\stackrel{0}{0}$ & $\stackrel{H}{-}$ & $\cong$ & $\underset{\sim}{\stackrel{0}{i}}$ & in & $\exists$ & $\stackrel{+}{-}$ & $\stackrel{\infty}{-}$ & ' & $\stackrel{\vartheta}{\sigma}$ & $\begin{array}{l}\text { Dे } \\
\text { है }\end{array}$ \\
\hline & \multirow{4}{*}{ 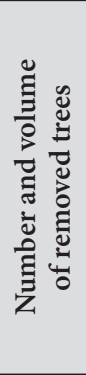 } & \multirow[b]{2}{*}{$D^{\prime}$} & $\alpha^{\circ}$ & $\stackrel{\circ}{-}$ & $\stackrel{9}{-}$ & $\stackrel{\infty}{\oplus}$ & $\begin{array}{l}0 \\
\infty\end{array}$ & ชె & $\stackrel{\vartheta}{\beth}$ & $\begin{array}{l}\stackrel{\circ}{ } \\
\text { }\end{array}$ & $\underset{r}{\ddot{r}}$ & $\stackrel{\sim}{\mathrm{N}}$ & $\begin{array}{l}\infty \\
\stackrel{\sim}{N}\end{array}$ & ' & \& & \\
\hline & & & 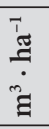 & $\stackrel{\stackrel{L}{n}}{0}$ & ఫे & $\stackrel{\infty}{\stackrel{\infty}{0}}$ & $\overrightarrow{\mathrm{N}}$ & $\stackrel{\infty}{\stackrel{\infty}{n}}$ & $\underset{+}{\overrightarrow{+}}$ & 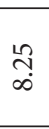 & ô & in & $\begin{array}{l}\infty \\
\infty \\
i n\end{array}$ & , & 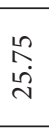 & 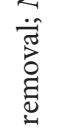 \\
\hline & & \multirow[b]{2}{*}{$z^{*}$} & $\alpha^{\circ}$ & $\hat{0}$ & 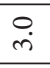 & $\hat{m}$ & $\infty$ & $\hat{a}$ & $\stackrel{\text { in }}{n}$ & $\begin{array}{l}\dot{0} \\
\ddot{n}\end{array}$ & $\hat{\sigma}$ & $\underset{\infty}{\infty}$ & $\stackrel{n}{0}$ & ' & $\stackrel{-}{\circ}$ & 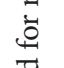 \\
\hline & & & 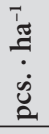 & - & 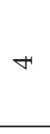 & in & $=$ & 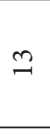 & $\vec{\sim}$ & \& & $a$ & $\exists$ & $\Xi$ & ' & $\stackrel{\text { }}{二}$ & 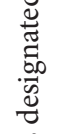 \\
\hline \multirow{6}{*}{ 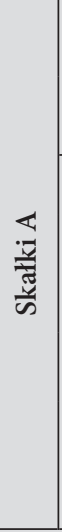 } & \multirow{2}{*}{ 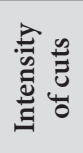 } & $\frac{1}{>}$ & $\partial^{\circ}$ & $\hat{i}$ & ' & $\stackrel{n}{\longrightarrow}$ & 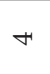 & ִָ & $\stackrel{+!}{0}$ & $\stackrel{n}{0}$ & $\hat{0}$ & ֻั & 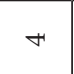 & ' & $\stackrel{\circ}{+}$ & $\underset{\Xi}{\mathscr{\Xi}}$ \\
\hline & & $\begin{array}{l}z \\
z\end{array}$ & $\partial^{\circ}$ & $\stackrel{n ?}{\longrightarrow}$ & ' & $\stackrel{-}{-}$ & $\stackrel{\infty}{\dot{m}}$ & $\hat{o}$ & $\hat{o}$ & $\stackrel{\circ}{-}$ & $\hat{\mathrm{i}}$ & $\stackrel{n ?}{\longrightarrow}$ & $\vec{m}$ & , & $\vec{\triangle}$ & छ \\
\hline & \multirow{4}{*}{ 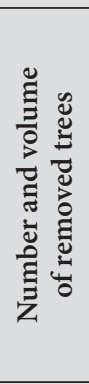 } & \multirow[b]{2}{*}{$\lambda^{\prime}$} & $\alpha^{\circ}$ & $\stackrel{\circ}{\circ}$ & ' & $\begin{array}{l}\infty \\
\stackrel{0}{0}\end{array}$ & $\begin{array}{l}\text { mo } \\
\stackrel{\sim}{\infty}\end{array}$ & $\cong$ & $\stackrel{\circ}{\dot{m}}$ & $\stackrel{n}{i}$ & กี & $\stackrel{b}{-}$ & $\underset{\stackrel{a}{\sim}}{a}$ & ' & 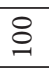 & อี \\
\hline & & & 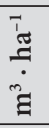 & $\begin{array}{l}\text { in } \\
\infty \\
\infty\end{array}$ & 1 & $\begin{array}{l}\infty \\
\infty \\
+\end{array}$ & 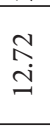 & $\begin{array}{l}\stackrel{0}{2} \\
\stackrel{0}{0}\end{array}$ & $\stackrel{m}{-}$ & $\stackrel{\circ}{=}$ & $\underset{\sim}{\sim}$ & $\begin{array}{l}\stackrel{+}{0} \\
\stackrel{0}{0}\end{array}$ & 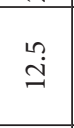 & , & $\begin{array}{l}\hat{\infty} \\
\dot{f} \\
\dot{f}\end{array}$ & $\begin{array}{l}\vec{z}^{=} \\
\dot{\overline{\tilde{F}}} \\
\overrightarrow{0}\end{array}$ \\
\hline & & \multirow[b]{2}{*}{$z^{z}$} & $\partial^{\circ}$ & $\stackrel{\circ}{a}$ & ' & $\stackrel{\circ}{\circ}$ & $\begin{array}{l}\stackrel{\circ}{\mathrm{i}} \\
\text { in }\end{array}$ & $\stackrel{\circ}{\circ}$ & 우․ & $\stackrel{0}{0}$ & $\stackrel{\circ}{\stackrel{1}{1}}$ & $\stackrel{\circ}{\circ}$ & $\begin{array}{l}0 \\
\infty \\
\infty\end{array}$ & , & 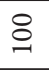 & \\
\hline & & & 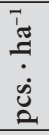 & $a$ & 1 & 으 & $\underset{\sim}{\sim}$ & 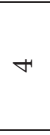 & in & 0 & $\bumpeq$ & $a$ & $\stackrel{\infty}{\sim}$ & ' & 8 & 苛 \\
\hline \multicolumn{4}{|c|}{$\frac{\frac{y}{y}}{\frac{\pi}{n}}$} & 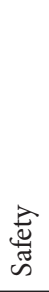 & 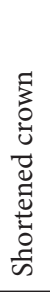 & 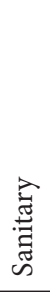 & 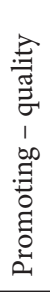 & 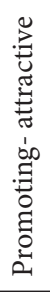 & 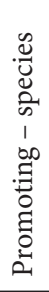 & $\begin{array}{l}0 \\
0 \\
0 \\
0 \\
00 \\
00 \\
0 \\
0 \\
0 \\
0 \\
0 \\
0\end{array}$ & 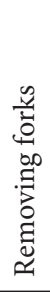 & 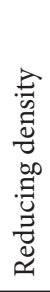 & 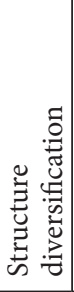 & 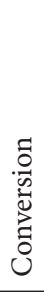 & 胥 & 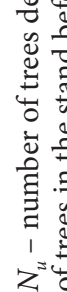 \\
\hline
\end{tabular}


measured by the number of removed trees, it ranged from approx. 17\% (Skałki A and Górka) to $20.2 \%$ (Skałki P). Among the tasks of cuts in the groups of Skałki A and Górka there were no conversion cuts; whereas this type cuts dominated in Skałki P - both in terms of the numbers of trees concerned (55.1\%) and in terms of the volume of trees (69.9\%). In terms of the number of trees and their thickness, in the Skałka A group, the highest number of trees for selection cuts was designated to promote specimens with higher trunk quality $(22.0 \%$ and $28.3 \%)$, followed by cuts leading to structural differentiation (18\% and $27.9 \%$ ). The share of cuts improving safety and sanitation cuts is also significant (Table 5).

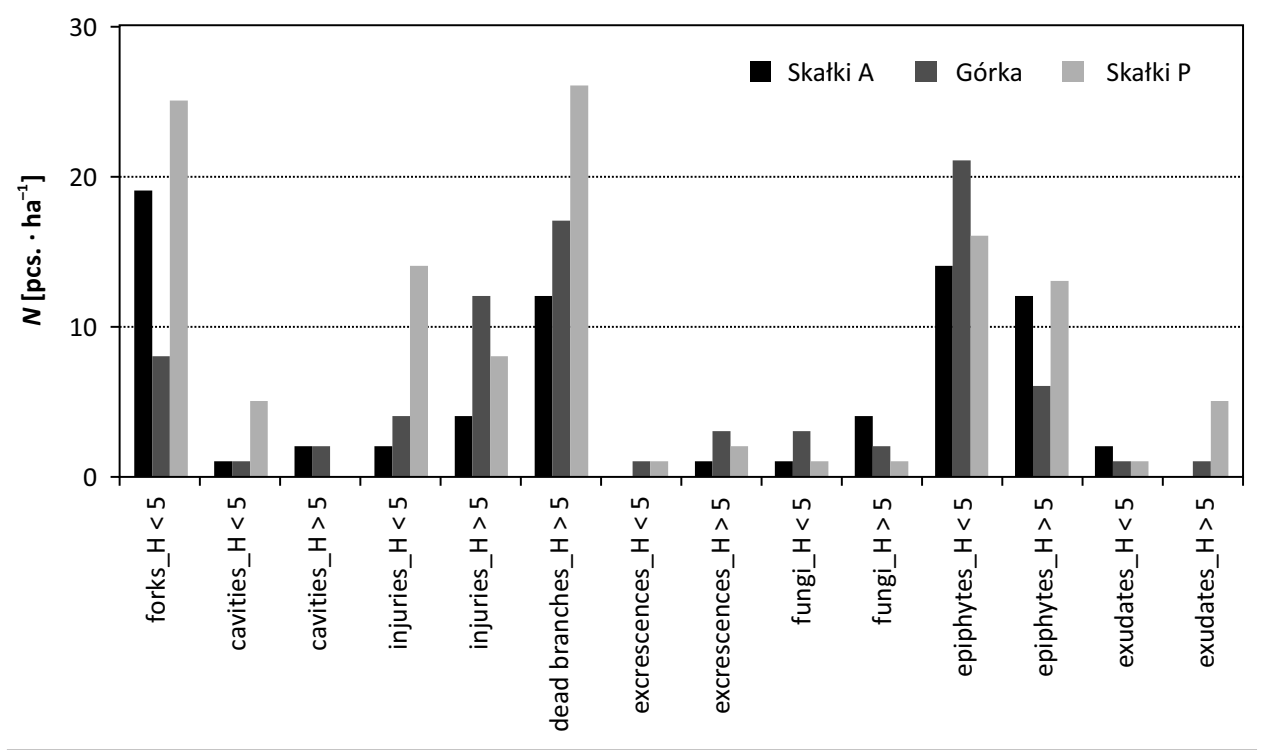

Fig. 2. The number $(N)$ of specific types of tree microhabitats

In the Górka group of tree stands, in terms of the number of trees and their volume, the task of supporting the groups was dominant (33.6\% and 32\%). The second highest number of cuts, in terms of the number of trees, comprised selection cuts promoting rare species $(15.7 \%)$, and in terms of volume, it was the cuts aimed at structural differentiation $(22.8 \%)$. A relatively large share was also found of the cuts promoting specimens that are attractive in terms of tree shape and with a higher trunk quality (Table 5).

In both groups of tree stands, no cuts were made to remove invasive species because no such species were found in the tree layer, whereas the presence of the American bird cherry in the undergrowth was low. 


\section{Discussion and conclusions}

The groups of stands covered by the present study differed in terms of species composition, assessment traits, species diversity indicators, and the purpose of cuts. These stands were created as a result of the afforestation of wastelands in the 1930s and 1940s (Dubiel et al. 2011) and are characterized by very high species richness (from 10 to 20 species in the group of stands), including a small but visible share of alien species (5 species). The species composition differs from the recommended types of tree stands (ZHL 2012) and specific replacement forest communities in the oak-hornbeam habitats (Dubiel and Szwagrzyk, eds. 2008). Compared to managed stands, a smaller share of valuable species such as English oak (Quercus robur L.), common beech (Fagus silvatica L.), and silver fir (Abies alba Mill.) was found, while species diversity is significantly greater, which should be considered an advantage of the forest stands in the studied area. Black pine (Pinus nigra J.F. Arnold) dominated in the group of stands where the species composition was recommended for transformation (in Skałki P), especially in terms of volume. The presence of alien species is an important research problem (FAO 2010).

The study does not take into account the task of removing invasive species, e.g. ash maple or American bird cherry, but the potential need for such cuts requires monitoring (Gazda 2012).

The found tree dimensions were low in relation to social preferences. Trees of considerable size are aesthetically valuable in urban forests. They also make the forest landscape more varied (Nielsen and Jensen 2007). Single specimens of extraordinary dimensions should be supported and made visible in the forest landscape.

The tree stands were dominated by two tree layers, whereas the lowest third layer was sparse. These stands show great potential in terms of structure differentiation, as the advanced regeneration is very abundant. The species composition of the saplings, however, is dominated by heliotropic species (Acer sp. maples), which survive in the lower layer only when intensive cuts are applied in short intervals. Shade-tolerant species have a smaller share. In the introduction to the paper, attention was drawn to the legitimacy of preferring complex vertical structures of stands in urban forests. In the 1970s and 1980s, cities in southern Sweden recommended the use of many different species of trees and shrubs to create complex structures as soon as possible after establishment, in order to promote recreation and biodiversity. Richnau et al. (2012) conducted an analysis of the effectiveness of this model of treatment. They found that frequently recurring thinning procedures are key to achieving complex structures. Increasing the number of species can increase resistance to neglect of treatments. However, rather than trying to maximize the total number of species, it makes more sense to focus on a few key species in each layer (Richnau et al. 2012). In this context, it is worth recommending supplementation of the species composition with such species as: silver fir (Abies alba Mill.), European beech (Fagus silvatica L.) or European yew (Taxus baccata L.). Most probably, the common hornbeam (Carpinus betulus L.) occurring in the undergrowth will increase its share over time. 
On the basis of the slenderness coefficient $(H / D)$ of trees and the high level of risk of dispersion to many species, the stands were identified as stable (Jelonek et al. 2013). The vitality of trees in combination with the length of the crown as an indicator of growth energy (Kaźmierczak 2012) also showed a satisfactory level, except for pine trees (Pinus sp.). The live crown ratio of pines amounted to $24-27 \%$, which points to their low vitality. Żybura (1987) indicates that Scots pines (P. silvestris) in older age classes should have the $L_{k} / H$ ratio of $33 \%$.

The found species diversity was higher than in the oak stands within the Płyta Krotoszyńska reserve, where the SHDI index was 0.35-0.97 (Szmyt 2017), while the registered species constitute only a part of the Polish dendroflora (Danielewicz and Wiatrowska 2015). Biodiversity rates were lower than in US urban forests (Blood et al. 2016). Reliable comparisons with other forests would require a unified taxonomic classification. The presence of tree microhabitats increases biodiversity. The larger the diameter of the tree, the more microhabitats were recorded (Spearman's correlation coefficient was 0.4 ), which is also indicated by the results published by Vuidot et al. (2011). In managed stands, specimens with a large number of niches are often removed (Bütler et al. 2013). Due to the positive impact on the aesthetics of the forest (Larrieu and Cabanettes 2012), these trees may be desirable in urban forests, also due to educational considerations.

The level of filling the space with regeneration should be considered satisfactory. The sustainability of the forest can be ensured by the presence of saplings (advanced regeneration) and a large number of young trees (7-34 cm thick). The species composition of the renewals was dominated by maples (Acer sp.), and similarly to the tree stand layer, it significantly differed from the recommended stand types in upland forest and fresh forest habitats (ZHL 2012). However, due to the dominant recreational function, changing the species composition is not necessary. More intensive efforts to release maples and other heliotropic species should be recommended, as well as endeavours to support all existing species. Supplementing the species composition, especially as part of conversion, with the aforementioned shade-tolerant conifers will revive the winter colour monotony of deciduous stands.

The abundant undergrowth should only be cut along the paths in order to improve transparency and safety (Ode and Fry 2002).

The cutting tasks differed between the particular groups of stands. Removal of specimens that threatened safety as well as sanitary cuts are routinely performed by ZZM Kraków near places of increased tourist traffic (paths, clearings). A helpful and simple measure in assessing the objectivity of the procedure was the VTA method (Szewczyk 2012). Apart from meeting the general assumptions of thinning, selective cuts were a tool that differentiated the forest landscape. In long-term planning, it would be useful to define the desired types of forest forms from the perspective of landscape architecture (Richnau et al. 2012). Generally, the trees in the study area were characterized by a low quality of the trunk. The cuts supporting a deficit of good-quality trees made up a significant share. Seemingly, this is a secondary criterion, as obtaining raw material in urban forests is of secondary importance (Zając et al. 2008). However, with the domi- 
nance of faulty tree shape traits, urban forests may give off an impression of being sick and neglected. Displaying trees with normal shape traits also has an educational value, as does promoting rare species (Edwards et al. 2012). Supporting trees with attractive shape significantly differs from the principles of thinning used in managed forests (Jaworski 2013). These are low-branched trees with spreading crowns, sometimes with deformed trunks, taking the form of the so-called speaders ("wolf" trees). However, they are characterized by a picturesque appearance and often a considerable thickness of trunks. These features have a positive effect on the recreational function of the forest. In the stands of Skałki Twardowskiego reserve there are suitable conditions for the release of the saplings and the lower layer, which is replacing older generations of trees. In Górka Pychowicka reserve, the development of stands, to a large extent, should be based on the promotion of tree biogroups. The release of monospecies groups has a positive effect on their vitality and stability (Zajączkowski 1994). The low vitality of pines and a significant shortening of crowns may in the near future cause their dieback in stands dominated by this species. Initiating the conversion process with the introduction of shade-tolerant species will contribute to diversifying the structure and architecture of the forest interior. It may also fit into the framework of one of the types of model stands (Nielsen and Jensen 2007). However, more intense cuts related to the transformation may be negatively perceived by the general public (Gundersen and Frivold 2008). Felling, clearing, and planting operations must be carried out very efficiently so that the debris remains in the area for as short a time as possible.

\section{Disclosure of funding}

This research was financed by the Ministry of Science and Higher Education of The Republic of Poland.

\section{References}

BDL. Bank Danych o Lasach. 2020. Mapa Interaktywna. Opis taksacyjny drzewostanu. https:// www.bdl.lasy.gov.pl/portal/mapy (accessed: 18.03.2020).

Blood A., Starr G., Escobedo F., Chappelka A. 2016. How Do Urban Forests Compare? Tree Diversity in Urban and Periurban Forests of the Southeastern US. Forests, 7, 120.

Bütler R., Lachat T., Larrieu L., Paillet Y. 2013. Habitat trees: Key elements for forest biodiversity. Integrative Approaches as an Opportunity for the Conservation of Forest Biodiversity. European Forest Institute, Joensuu, 84-91.

Czuraj M. 1998. Tablice miąższości kłód odziomkowych i drzew stojących. Oficyna Edytorska „Wydawnictwo Świat”, Warszawa.

Danielewicz W., Wiatrowska B. 2015. Różnorodność i przemiany dendroflory Polski. Studia i Materiały CEPL w Rogowie, 17, 42/1: 13-26.

Dubiel E., Nobis A., Nobis M. 2011. Vascular plants and plant communities of the Zakrzowek district (Krakow). Fragmenta Floristica et Geobotanica Polonica, 18(1): 47-81.

Dubiel E., Szwagrzyk J. (red.) 2008. Atlas roślinności rzeczywistej Krakowa. UMK, Kraków, 159. 
Edwards D., Jay M., Jensen F.S., Lucas B., Marzano M., Montagné C., Peace A., Weiss G. 2012. Public preferences for structural attributes of forests: Towards a pan-European perspective. Forest Policy and Economics, 19: 12-19.

Eggers J., Lindhagen A., Lind T., Lämås T., Öhman K. 2018. Balancing landscape-level forest management between recreation and wood production. Urban Forestry and Urban Greening, 33: $1-11$.

FAO. 2010. Global forest resources assessment. 2010 - Main report. FAO Forestry Paper 163. Rome, Italy.

Gazda A. 2012. Stan badań nad obcymi gatunkami drzew w polskich lasach. Studia i Materiały CEPL w Rogowie, 14, 33, 4: 44-52.

Geoportal 2. 2020. Mapa topograficzna i lotnicza. https://mapy.geoportal.gov.pl/imap/Imgp_2. html (accessed: 18.03.2020).

Gerstenberg T., Hofmann M. 2016. Perception and preference of trees: A psychological contribution to tree species selection in urban areas. Urban Forestry and Urban Greening, 15: 103-111.

Giergiczny M., Czajkowski M., Żylicz T., Angelstam P. 2015. Choice experiment assessment of public preferences for forest structural attributes. Ecological Economics, 119: 8-23.

Gołos P. 2018. Społeczne i ekonomiczne aspekty pozaprodukcyjnych funkcji lasu i gospodarki leśnej - wyniki badań opinii społecznej. Prace IBL, 22. Sękocin Stary.

Grzelak-Kostulska E., Hołowiecka B. 2013. Lasy jako miejsca realizacji indywidualnych potrzeb aktywności i wypoczynku ludności. Studia i Materiały CEPL w Rogowie, 15, 37, 4: 104-110.

Gundersen V.S., Frivold L.H. 2008. Public preferences for forest structures: a review of quantitative surveys from Finland, Norway and Sweden. Urban Forestry and Urban Greening, 7: 241-258.

IUL. Instrukcja urządzania lasu. 2012. Część I. Instrukcja sporządzania projektu planu urządzenia lasu dla nadleśnictwa. PGL LP, CILP, Warszawa, $\$ 31$ ust. 15.

Jaszczak R., Wajchman S. 2015. Wybrane aspekty gospodarki leśnej w lasach miejskich Poznania i w Lasach Państwowych. Sylwan, 159(2): 160-167.

Jaszczak R., Ważyński B., Wajchman-Świtalska S. 2017. Prawne aspekty leśnictwa miejskiego w Polsce. Sylwan, 161(8): 659-668.

Jaworski A. 2013. Hodowla lasu. Tom II. Pielegnowanie lasu. PWRiL, Warszawa.

Jelonek T., Walkowiak R., Jakubowski M., Tomczak A. 2013. Wskaźniki stabilności drzew w drzewostanach sosnowych uszkodzonych przez wiatr. Sylwan, 157(5): 323-329.

Johnston M. 1996. A brief history of urban forestry in the United States. Arboricultural Journal, 20: $257-278$.

Kaliszewski A. 2006. Lasy miejskie - przeglad wybranych zagadnień na podstawie literatury. Leśne Prace Badawcze, 1: 103-118.

Kaźmierczak K. 2012. Przestrzeń wzrostu sosny w 35-letnim drzewostanie na przykładzie wybranych miar przestrzeni wzrostu pojedynczego drzewa. Sylwan, 156, 4: 280-286.

Korpel S., Penaz J., Saniga M., Tesar V. 1991 Pestovanie lesa. Priroda, Bratislava, Slovakia.

Kostecka J., Mazur-Pączka A., Jasińska T., Batóg K. 2012. Pojęcie „świadczenia ekosystemowe” i jego rola w edukacji dla zrównoważonego rozwoju (na przykładzie bzu czarnego Sambucus nigra L.). Inżynieria i Ochrona Środowiska, 15(4): 405-417.

Larrieu L., Cabanettes A. 2012. Species, live status, and diameter are important tree features for diversity and abundance of tree microhabitats in subnatural montane beech-fir forests. Canadian Journal of Forest Research, 42, 8: 1433-1445.

Larrieu L., Paillet Y., Winter S., Bütler R., Kraus D., Krumm F., Lachat T., Michel A., Regnery B., Vandekerkhove K. 2018. Tree related microhabitats in temperate and Mediterranean European forests: A hierarchical typology for inventory standarization. Ecological Indicators, 84: 194-207. 
Lawrence H.W. 1995. Changing Forms and Persistent Values: Historical Perspectives on the Urban Forest. In: Urban Forest Landscapes: Integrating Multidisciplinary Perspectives. Ed. G. Bradley. University of Washington Press, Seattle and London, 17-40.

Łonkiewicz B. 1997. Urządzanie i zagospodarowanie lasu $w$ terenach zurbanizowanych i uzdrowiskowych. Postępy Techniki w Leśnictwie, 64: 31-37.

MacDonald E., Mochan S., Connolly T. 2009. Validation of a stem straightness scoring system for Sitka spruce (Picea sitchensis (Bong.) Carr.). Forestry: An International Journal of Forest Research, 82, 4: 419-429.

Matuszko D., Piotrowicz K. 2018. Związek usłonecznienia i temperatury powietrza na podstawie wieloletniej serii klimatologicznej w Krakowie (1884-2016). Polskie Towarzystwa Geofizyczne, Komitet Geofizyki PAN. Przegląd Geofizyczny, 1-2: 15-29.

Motyka J., Czop M., Polak K. 2003. Wpływ warunków wodnych w kamieniołomie „Zakrzówek” w Krakowie na jego funkcję rekreacyjna. W: Kształtowanie krajobrazu terenów poeksploatacyjnych w górnictwie. Międzynarodowa Konferencja Naukowa, 10-12 grudnia 2003. Kraków, 208-219.

Nagendra H. 2002. Opposite trends in response for the Shannon and Simpson indices of landscape diversity. Applied Geography, 22: 175-186.

Nalepa S., Lach J., Murzynowski K., Soboń Ł., Górniak J., Jop M., Gałyga H. 2018. Powiatowy Program Zwiększenia Lesistości Miasta Krakowa na lata 2018-2040 (projekt). BULiGL, Kraków.

Nielsen A.B., Jensen R.B. 2007. Some visual aspects of planting design and silviculture across contemporary forest management paradigms - Perspectives for urban afforestation. Urban Forestry and Urban Greening, 6: 143-158.

Ode Å., Fry G. 2002. Visual aspects in urban woodland management. Urban For. Urban Greening, 1: 15-24.

PUL 2018. Plan Urządzenia Lasu na okres od 1 stycznia 2018 r. do 31 grudnia 2027 r. Grunty Skarbu Państwa użytkowane przez ZZM w Krakowie. Krameko sp. z. o. o., Kraków

Richnau G., Wiström B., Nielsen A.B., Löf M. 2012. Creation of multi-layered canopy structures in young oak-dominated urban woodlands - The 'ecological approach' revisited. Urban Forestry and Urban Greening, 11: 147-158.

Rutkowski J. 1992. Szczegółowa mapa geologiczna Polski 1:50 000. Arkusz 973 Kraków. Państwowy Instytut Geologiczny, Warszawa. Arkusz pobrano z CBDG.

Sienkiewicz J. 2010. Koncepcje bioróżnorodności - ich wymiary $i$ miary w świetle literatury. Ochrona Środowiska i Zasobów Naturalnych, 45: 7-29.

Szewczyk G. 2012. Arborystyka. Wybrane zagadnienia pielegnacji drzew. Wydawnictwo Uniwersytetu Rolniczego, Kraków, 130.

Szmyt J. 2017. Zróżnicowanie strukturalne wybranych drzewostanów dębu szypułkowego (Quercus robur L.) występujacych na terenie Płyty Krotoszyńskiej. Leśne Prace Badawcze, 78, 1: 14-27.

Vuidot A., Paillet Y., Archaux F., Gosselin F. 2011. Influence of tree characteristics and forest management on tree microhabitats. Biological Conservation, 144: 441-450.

Wajchman S. 2013. Rekreacyjne zagospodarowanie lasów miejskich miasta Poznania. Studia i Materiały CEPL w Rogowie, 15, 35: 119-126.

Ważyński B. 2007. Zasady prowadzenia gospodarki leśnej wokół aglomeracji miejskich. Biblioteczka leśniczego, 253. Wydawnictwo Świat, Warszawa.

Ważyński B. 2011. Urządzanie i rekreacyjne zagospodarowanie lasu. Poradnik leśnika. PWRiL, Warszawa.

Zając S., Gołos P., Głaz J., Kaliszewski A., Sikora A., Hildebrand K. 2008. Opracowanie metody delimitacji funkcji lasu oraz zasad wielofunkcyjnej i zrównoważonej gospodarki leśnej na przykładzie LKP Lasy Warszawskie. Dokumentacja naukowa IBL, etap II. 
Zajączkowski J. 1994. Biogrupy drzew w drzewostanach - możliwości i celowość ich wykorzystania przy prowadzeniu trzebieży. Prace IBL A, 778: 1-38.

ZHL. Zasady hodowli lasu. 2012. CILP, Warszawa.

Zygmunt R., Banaś J., Zięba S. 2014. Trwałość lasów miejskich na przykładzie "Lasu Wolskiego" w Krakowie. Studia i Materiały CEPL w Rogowie, 16, 39, 2A: 109-119.

Żybura H. 1987. Relation of the crown length of pine trees to the age and site quality of stand to the biosocial structure of trees. Annals of Warsaw Agricultural University SGGW-AR, 36: 61-68.

\section{CHARAKTERYSTYKA DRZEWOSTANÓW ORAZ ZADANIA CIĘĆ PIELĘGNACYJNYCH W LASACH MIEJSKICH NA PRZYKŁADZIE UROCZYSK SKAŁKI TWARDOWSKIEGO I GÓRKA PYCHOWICKA}

\section{STRESZCZENIE}

Celami pracy były: (1) ocena zasobów drzewnych, podrostów i podszytów; (2) inwentaryzacja mikrosiedlisk nadrzewnych oraz (3) określenie zadań cięć w atrakcyjnych z rekreacyjnego punktu widzenia drzewostanach Uroczysk Skałki Twardowskiego i Górka Pychowicka. Lokalizacja i skład gatunkowy przyszłego drzewostanu były kryteriami podziału na 3 grupy drzewostanów: Skałki Twardowskiego (Skałki A) - zachowanie składu gatunkowego; Górka Pychowicka - zachowanie składu gatunkowego (Górka); Skałki Twardowskiego - przebudowa składu gatunkowego (Skałki P). Praca stanowi studium przypadku, w którym na podstawie identyfikacji potrzeb określono zadania cięć pielęgnacyjnych w badanych obiektach. W pracy przedstawiono analizę struktury cięć pielęgnacyjnych realizujących następujące zadania: usunięcie drzew zagrażających bezpieczeństwu; usunięcie drzew z ekstremalnie skróconą koroną, silnie wysmuklonych; sanitarne - usunięcie drzew zamierających lub pozostawienie jako źródła mikrosiedlisk nadrzewnych; usunięcie gatunków inwazyjnych, takich jak np. klon jesionolistny, czeremcha amerykańska; selekcja pozytywna (na korzyść cennych jakościowo, efektownych pokrojowo, rzadkich gatunków); popieranie jednogatunkowych grup drzew (usunięcie innego gatunku, uwolnienie grupy); usunięcie jednego pnia u drzew rozwidlonych w odziomku ( $\leq$ 1,3 m wysokości); zmniejszanie zagęszczenia; popieranie dolnego piętra i podrostu (kształtowanie zróżnicowanej struktury); cięcia przebudowy; usunięcie podszytu, podrostu, dolnego piętra, dla poprawy widoczności w odległości $20 \mathrm{~m}$ od ścieżek pieszych i polan. Grupy drzewostanów objęte opracowaniem różniły się pod względem składu gatunkowego, cech taksacyjnych, wskaźników różnorodności gatunkowej i zadań wyznaczanych cięć. Drzewostany charakteryzują się bardzo dużym bogactwem gatunkowym (od 10 do 20 gatunków w grupie drzewostanów), w tym niewielkim, ale widocznym udziałem gatunków obcych (5 gatunków) (tab. 1). Stwierdzone dymensje drzew były niskie w stosunku do preferencji społecznych (ryc. 1). W opisywanych drzewostanach dominowały dwie warstwy drzew, najniższa trzecia warstwa była reprezentowana nielicznie (tab. 2). Drzewostany te wykazują jednak duży potencjał pod względem różnicowania struktury, gdyż podrost jest bardzo liczny (tab. 3). Często powtarzające się przerzedzenia 
są kluczowe dla uzyskania złożonych struktur. Skład gatunkowy odnowień był zdominowany przez klony (tab. 3). Jednak z racji dominującej funkcji rekreacyjnej zmiana składu gatunkowego nie wydaje się konieczna. Należy zalecić intensywniejsze zabiegi uwalniające klony i inne gatunki światłożądne oraz popieranie wszystkich istniejących gatunków. Warto zalecić uzupełnianie składu gatunkowego o takie gatunki jak jodła, buk czy cis. Domieszka gatunków iglastych ożywi zimową monotonię kolorystyczną drzewostanów liściastych. Występujący w podroście grab prawdopodobnie z czasem uzyska większy udział. Stwierdzona różnorodność gatunkowa jest bardzo wysoka (tab. 2). Występowanie nadrzewnych mikrosiedlisk dodatkowo zwiększa bioróżnorodność. W lasach miejskich te drzewa mogą być pożądane również ze względów edukacyjnych (ryc. 2). Obficie występująca warstwa podszytowa (tab. 4) powinna być poddawana cięciom jedynie przy ścieżkach, w celu poprawy przejrzystości i bezpieczeństwa. Cięcia przebudowy dominowały w drzewostanach grupy Skałki P. W grupie Skałki A promowano głównie osobniki o wyższej jakości pnia i wyznaczono cięcia prowadzące do różnicowania struktury. Zwraca również uwagę duży udział cięć poprawiających bezpieczeństwo oraz sanitarnych. W grupie drzewostanów Górka dominowały: zadanie popierania grup, selekcja promująca rzadszy gatunek i cięcia różnicujące strukturę. Stosunkowo duży udział miały również cięcia promujące osobniki efektowne pod względem pokroju oraz o wyższej jakości pnia (tab. 5).

\section{SŁOWA KLUCZOWE}

urządzanie lasu • rekreacja • hodowla lasu • trzebieże • leśnictwo miejskie 